\title{
Estudio clínico-patológico sobre el cáncer de próstata incidental en pacientes intervenidos bajo el supuesto diagnóstico de HBP sintomática
}

\author{
E. Fernández Rosado, F. Gómez Veiga, L. Álvarez Castelo, M. Ruibal Moldes, V. Chantada Abal, \\ M. González Martín
}

Servicio de Urología. Complejo Hospitalario Universitario Juan Canalejo. La Coruña.

Actas Urol Esp 2006; 30 (1): 33-37

\begin{abstract}
RESUMEN
ESTUDIO CLÍNICO-PATOLÓGICO SOBRE EL CÁNCER DE PRÓSTATA INCIDENTAL EN PACIENTES INTERVENIDOS BAJO EL SUPUESTO DIAGNÓSTICO DE HBP SINTOMÁTICA

Objetivo-Introducción: Estudio retrospectivo de una serie de pacientes intervenidos mediante adenomectomía prostática suprapúbica (APS) y resección transuretral prostática (RTU-P) bajo el supuesto diagnóstico de HBP sintomática no respondedora al tratamiento médico conservador. Análisis de la prevalencia, incidencia, datos clínico-patológicos, tratamiento, progresión tumoral y evolución de los pacientes con cáncer de próstata incidental (CPI) detectado.

Material y métodos: Se revisaron 1.593 pacientes intervenidos durante 6 años consecutivos (1996-2001). APS 35\%, RTUP 65\%. Revisión de todas las anatomías patológicas de los especimenes quirúrgicos y de las evoluciones de los pacientes con CPI.

Resultados: 78 CPI; Prevalencia 4,89\%; Incidencia 13 casos/año. Edad media 73,6 años. Tacto rectal normal en todos, PSA medio $6 \mathrm{ng} / \mathrm{ml}$ (0,5-30). Grupo APS: 25 CPI (32\%); prevalencia 4,55\%; incidencia 4 casos/año; PSA medio 7,7 ng/ml (2,8-30); volumen enucleado medio 65 gs. Grupo RTU-P: 53 CPI (68\%); prevalencia 5,07\%; incidencia 9 casos/año; PSA medio $5,2 \mathrm{ng} / \mathrm{ml}(0,5-29)$; volumen resecado medio $20 \mathrm{gs} .22 \%$ biopsiados previamente por PSA elevado medio de $14 \mathrm{ng} / \mathrm{ml}$ (4,8-30). Mediana de Gleason 5 (media 4,8), rango 3-8. pTla 66\%, pT1b 33\%. Tratamiento: 57\% seguimiento vigilado sin tratamiento; $18 \%$ tratamiento hormonal; 3\% finasteride; 9\% Prostatectomía Radical; 9\% Radioterapia. Pérdidas 4\%. Seguimiento medio 47,19 meses (12-96). Progresión tumoral 13,3\% (10 pacientes). Mortalidad CPI específica $2,6 \%$ (2 pacientes).

Conclusiones: No observamos diferencias significativas entre la prevalencia de CPI en los dos grupos (APS y RTU-P). Los tumores detectados fueron mayoritariamente bien diferenciados y en estadio pTla. En más de la mitad de los casos se decidió una actitud expectante sin tratamiento (57\%). 13,3\% de progresión tumoral tras 47,19 meses de seguimiento medio y mortalidad CPI específica del 2,6\%.
\end{abstract}

Palabras clave: Cáncer. Próstata, Incidental.

\section{RESUMEN}

CLINICOPATHOLOGICAL STUDY OF INCIDENTAL CANCER PROSTATE IN PATIENTS UNDERGOING SURGERY FOR SYMPTOMATIC DIAGNOSIS OF BPH

Objectives-Introduction: Retrospective study of a series of patients with prostate surgery (suprapubic prostate adenomectomy -APS-, and prostate transurethral resection -RTU-P- ) for presumed BPH symptomatic non-respondent to the conservative medical treatment. Analysis of prevalence, incidence, clinical-pathological, treatment, tumor progression and evolution of the patients with incidental prostate cancer (CPI) detected.

Material and methods: 1593 patients with prostate surgery (APS and RTU-P) during 6 years (1996-2001) were revised. APS 35\%, RTU-P 65\%. Revision of all pathological anatomy of surgical specimens and the evolutions of the patients with CPI.

Results: 78 CPI; Prevalence 4,89\%; Incidence 13 cases/year. Mean age 73.6 years. Digital rectal examination was normal in 100\%, mean PSA $6 \mathrm{ng} / \mathrm{ml}$ (0.5-30). Group APS: 25 CPI (32\%); prevalence 4.55\%; incidence 4 cases/year; mean PSA $7.7 \mathrm{ng} / \mathrm{ml}$ (2.8-30); mean weight resection 65 gs. Group RTU-P: 53 CPI (68\%); prevalence 5,07\%; incidence 9 cases/year; mean PSA $5.2 \mathrm{ng} / \mathrm{ml}(0,5-29)$; mean weight resection 20 gs. $22 \%$ biopsy previously by high PSA, mean PSA $14 \mathrm{ng} / \mathrm{ml}(4,8-$ 30). Gleason average 5 (mean 4.8), rank 3-8. pTla 66\%, pT1b 33\%. Treatment: $57 \%$ follow-up watched without treatment (wait and see); 18\% hormonal treatment; 3\% finasteride; 9\% Radical Prostatectomy; 9\% radiotherapy. Follow Lost 4\%. Mean follow-up 47.19 months (12-96). Tumor progression 13.3\% (10 patients). Specific CPI mortality $2.6 \%$ (2 patients).

Conclusions: We didn't observe significant differences between the prevalence of CPI in both groups (APS and RTU-P). The detected tumours were mainly well differentiated and in stage pTla. In more than half of the cases an expectant attitude without treatment was decided. $13,3 \%$ of tumor progression after 47.19 months of follow mean and specific CPI mortality $2.6 \%$.

Keywords: Cancer. Prostate. Incidental. 
$\mathrm{E}$ n los últimos años, principalmente como consecuencia de la introducción del PSA, se ha producido un cambio significativo en la presentación del cáncer prostático. Tradicionalmente, los pacientes se diagnosticaban en etapas avanzadas y demasiado tarde para las terapias curativas disponibles. Progresivamente hasta la actualidad, el cáncer prostático ha comenzado a detectarse en pacientes asintomáticos y con enfermedad localizada, motivando importantes controversias y alternativas para su manejo. En la mayoría de los pacientes con síntomas (grupo cada vez más reducido pero aún considerable) la enfermedad no se encuentra confinada al órgano. Estos pacientes se presentan con manifestaciones asociadas a la enfermedad diseminada (síndrome general, anemia, dolores óseos) o debido a la afectación local de la misma (hematuria, retención urinaria, fracaso renal con compromiso obstructivo).

Un grupo poblacional interesante lo forman los pacientes con síntomas urinarios "obstructivos" (secundarios a una supuesta HBP sintomática) y que no presentan anormalidades sospechosas al tacto rectal, e incluso tienen valores normales de PSA o han sido biopsiados por presentar alteración en el tacto rectal o en el PSA. Hasta en un $10-20 \%$ de estos pacientes se encuentra un cáncer de próstata incidental (pTla o pT1b) cuando son sometidos a tratamiento quirúrgico por una supuesta hiperplasia prostática benigna (HBP) ${ }^{1-5}$.

El cáncer de próstata incidental (CPI), insignificante o irrelevante (también conocido como cáncer de próstata latente), es una forma de cáncer prostático que no tiene significado clínico en el paciente en el que fue descubierto. El carcinoma incidental (sin manifestaciones clínicas, silente, descubierto casualmente en el examen microscópico de tejido prostático resecado bajo el diagnóstico de enfermedad no maligna) y el latente (descubierto en autopsias) parecen tener una frecuencia mucho mayor que el carcinoma manifiesto y el oculto. Por lo tanto, aparentemente existe sólo un pequeño porcentaje de cánceres que progresan y se diseminan. El porqué de esta agresividad selectiva se desconoce por completo.

El objetivo de este estudio retrospectivo ha sido el de revisar una serie de pacientes interve- nidos mediante adenomectomía prostática suprapúbica (APS) y resección transuretral prostática (RTU-P) bajo el supuesto diagnóstico de HBP sintomática no respondedora al tratamiento médico conservador. Posteriormente se analizaron los resultados para el estudio de la prevalencia, incidencia, datos clínico-patológicos, tratamiento y evolución del cáncer de próstata incidental (CPI) detectado.

\section{MATERIAL Y MÉTODOS}

Se revisaron retrospectivamente (fin de la revisión para el seguimiento de los casos en enero del 2004), 1.593 pacientes intervenidos durante 6 años consecutivos (1996-2001). Los criterios de selección fueron: pacientes con supuesta HBP sintomática, sin respuesta satisfactoria al tratamiento farmacológico conservador, candidatos a tratamiento quirúrgico y operados durante el periodo seleccionado para el estudio. De ellos, fueron APS el 35\% (549 pacientes), y RTU-P el $65 \%$ (1.044). Fueron excluidos del estudio RTU-P en pacientes con cáncer de próstata conocido previamente, otras técnicas quirúrgicas sin muestra de tejido prostático analizable como la incisión transuretral prostática (ITUP) sobre mínimos adenomas obstructivos, electrofulguraciones prostáticas, y otras RTU-P funcionalespaliativas en pacientes con muy alta sospecha de cáncer prostático pero sin confirmación histológica hasta el momento.

Todos los informes de las anatomías patológicas de los especímenes quirúrgicos fueron revisados, especialmente los casos con CPI detectados, así como los tratamientos y las evoluciones de los pacientes con CPI.

\section{RESULTADOS}

De los 1.593 pacientes intervenidos por supuesta HBP sintomática, en 78 de ellos se detectó un CPI (Prevalencia 4,89\%; Incidencia 13 casos/año). La edad media de los pacientes con CPI fue de 73,6 años, y el tacto rectal (TR) normal en todos. El PSA medio de los pacientes con CPI fue de $6 \mathrm{ng} / \mathrm{ml}(0,5-30)$. Por grupos de pacientes, distinguimos el grupo de los operados de HBP mediante cirugía abierta (APS) y los operados por vía endoscópica (RTU-P) (Tabla 1). 
De todos los casos de CPI (78), 17 (21,8\%) fueron biopsiados previamente a la cirugia por PSA elevado, con una media de $14 \mathrm{ng} / \mathrm{ml}(4,8-30)$. Como ya se comentó, el TR era normal en todos los casos (biopsiados y no biopsiados). La mediana del Gleason de todos los casos de CPI fue de 5 (media 4,8 ), con un rango entre 3-8 y una moda de 4 . Por estadios de la UICC, el 66,6\% (52 casos, 22 del grupo APS y 30 del grupo RTU-P) correspondieron a un pTla (con volúmenes tumorales inferiores al $5 \%$ del tejido enucleado o resecado), y el 33,3\% (26 casos, 3 del grupo APS y 23 del grupo RTU-P) a un pT1b (mayores al 5\%) (Tabla 2).

Respecto al tratamiento empleado para el manejo de los CPI (78), 3 casos se perdieron $(3,84 \%)$ tras el diagnóstico de CPI, con lo que no

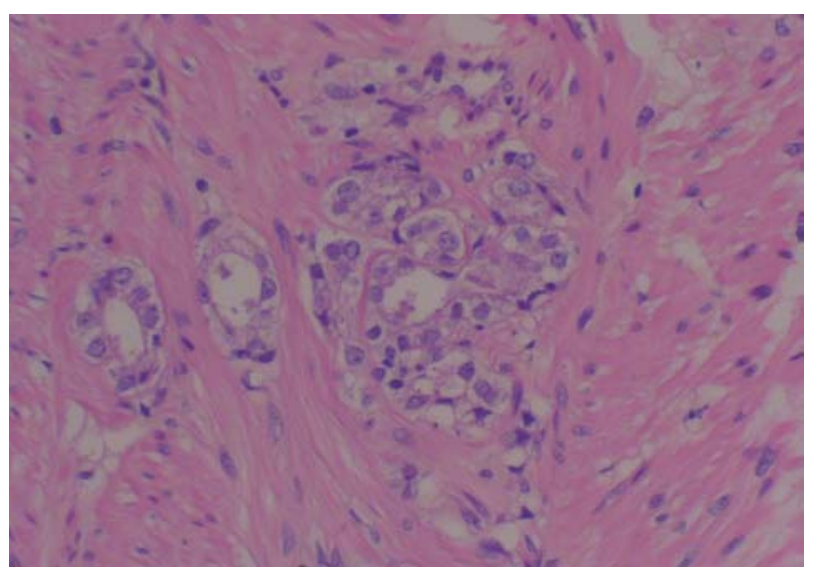

FIGURA 1. Foco de adenocarcinoma prostático detectado mediante biopsia durante el seguimiento de un paciente con cáncer de próstata incidental (progresión tumoral). Se observan nucleolos evidentes y glándulas fusionadas entre si (20x).

\section{Tabla 1}

Resultados de la casuística de Cáncer de Próstata Incidental (CPI) en pacientes intervenidos de supuesta HBP sintomática según el tipo de cirugía: adenomectomía prostática suprapúbica (APS) o resección transuretral prostática (RTU-P)

\begin{tabular}{lccc}
\hline & APS & RTU-P & Total \\
\hline n CPI & $25(32 \%)$ & $53(68 \%)$ & $78(100 \%)$ \\
Prevalencia & $4,55 \%$ & $5,07 \%$ & $4,89 \%$ \\
Incidencia (casos/año) & 4 & 9 & 13 \\
Edad media (años) & $75,2(63-90)$ & $72,2(58-85)$ & $73,6(58-90)$ \\
PSA medio (ng/ml) & $7,7(2,8-30)$ & $5,2(0,5-29)$ & $6(0,5-30)$ \\
Volumen enucleado medio de & & & $34,4(4-159)$ \\
los casos con CPI (gramos) & 65 & 20 & \\
\hline
\end{tabular}

\section{Tabla 2}

Cuadro resumen y distributivo de casos de Cáncer de Próstata Incidental (CPI) según prevalencia, estadio, pérdidas para el seguimiento, tratamiento, progresión tumoral y mortalidad

\begin{tabular}{lccc}
\hline & APS & RTU-P & Totales \\
\hline n (CPI) & $25 / 549(4,5 \%)$ & $53 / 1044(5 \%)$ & $78 / 1593(4,8 \%)$ \\
pTla & $88 \%(22 / 25)$ & $56,6 \%(30 / 53)$ & $66,6 \%(52 / 78)$ \\
pT1b & $12 \%(2 / 25)$ & $43,4 \%(23 / 53)$ & $33,3 \%(26 / 78)$ \\
Pérdidas (para seguimiento) & 0 & 3 & $75(78-3)$ \\
Sin tratamiento (vigilancia) & 21 & 24 & $45(57,7 \%)$ \\
Tto. hormonal & 2 & 12 & $14(18 \%)$ \\
Finasteride & 2 & 0 & $2(2,5 \%)$ \\
Prostatectomía radical & 0 & 7 & $7(9 \%)$ \\
Radioterapia & 0 & 7 & $7(9 \%)$ \\
Progresión tumoral & $12 \%(3 / 25)$ & $14 \%(7 / 50)$ & $13,3 \%(10 / 75)$ \\
Progresión tumoral grupo & & & \\
sin tto. & $3 / 21(14,2 \%)$ & $4 / 24(16,6 \%)$ & $7 / 45(15,5 \%)$ \\
Progresión tumoral grupo & & & \\
tto. hormonal & 0 & $3 / 12(25 \%)$ & $3 / 14(21,4 \%)$ \\
Mortalidad CPI específica & 0 & $4 \%(2 / 50)$ & $2,6 \%(2 / 75)$ \\
\hline
\end{tabular}

fueron tratados ni seguidos posteriormente en nuestro centro. Del resto de los casos (75), el 57,7\% (45 pacientes) recibieron un seguimiento vigilado estrecho sin tratamiento (esperar y ver); el 17,9\% (14 pacientes) recibieron algún tipo de tratamiento hormonal (en sus distintas variantes); el $2,56 \%$ (2 pacientes) se trataron con finasteride; el $8,97 \%$ (7 pacientes) fueron operados mediante una Prostatectomía Radical (PR); y el 8,97\% (7 pacientes) recibieron Radioterapia externa (RT) (Tabla 2). El seguimiento medio (con unas pérdidas para seguimiento, 3 pacientes, inferiores al 4\%) hasta la fecha del estudio fue de 47,19 meses (rango 12-96).

En un total de 10 pacientes de los 75 con CPI tratados y seguidos en nuestro centro se detectó progresión tumoral (13,3\%). De estos, 3 eran del grupo de los operados mediante APS (3 de 25, 12\% de progresión tumoral) y 7 del de las RTU-P (7 de 50, 14\% de progresión tumoral). El tiempo medio en estos pacientes hasta la progresión fue de 37,3 meses (rango 184 meses). Los grupos de trata- 
miento a los que pertenecían los pacientes en los que se detectó progresión tumoral (10 pacientes) eran: seguimiento vigilado estrecho sin tratamiento 7 casos, tratamiento hormonal 3 casos. La detección de la progresión tumoral se confirmó mediante biopsia transrectal (Fig. 1), indicada por elevación del PSA y por alta sospecha de tumor residual en dos casos que fueron biopsiados precozmente. El TR no fue valorable para el seguimiento y detección de las progresiones tumorales, ya que su interpretación se encuentra artefactada por la cirugía previa reciente. El grado de Gleason se elevó al detectar las progresiones tumorales en un rango entre 0 y 4 puntos (elevación media de 1,5 puntos con respecto al Gleason de la pieza enucleada o resecada en la APS o RTU-P en donde se detectó el CPI).

Respecto a los casos en los que se realizó una PR (7 casos) como tratamiento al CPI detectado, todos eran pacientes del grupo de los operados mediante RTU-P, con una edad media de 65,5 años (inferior a la edad media del grupo global de los CPI que era de 73,6 años). El tiempo de espera medio entre la detección del CPI y la PR fue de 7,2 meses (rango 2-13 meses). La mediana del grado de Gleason del CPI detectado en este grupo fue de 5 (media 5,1). La mediana del grado de Gleason de la pieza quirúrgica tras la PR fue de 5 (media 4,2 debido a que en un caso no se detectó tumor residual, pT0). En los casos en que si se detectó tumor residual (6 de los 7 casos), el Gleason fue el mismo que el del CPI detectado incidentalmente, excepto en un caso en que fue un punto menor. Respecto a los estadios tumorales del CPI en este grupo, 2 casos eran pTla y 5 casos pT1b. Los estadios de la pieza quirúrgica de la PR fueron: 2 casos pT2a; 4 casos pT2b; y un pTO. La evolución fue favorable en todos ellos tras una media de seguimiento tras la PR de 55,7 meses (rango 36-83). Ningún paciente al que se le realizó una $\mathrm{PR}$ progresó posteriormente durante el periodo de seguimiento.

Respecto a los casos en los que se realizó RT externa (7) como tratamiento al CPI detectado, todos eran pacientes del grupo de los operados mediante RTU-P, con una edad media de 70,4 años, edad intermedia entre la edad media de los que se trataron mediante PR (65,5 años) y la del grupo global de los CPI (73,6 años). El tiempo de espera medio entre la detección del CPI y la RT fue de 8,1 meses (rango 4-16 meses). Un paciente del grupo de las APS y otro del grupo de las RTU-P que progresaron fueron tratados posteriormente también con RT externa, y otro del grupo de las RTUP que progresó a los 7 años de seguimiento se trató con braquiterapia de baja tasa. La mediana del grado de Gleason del CPI detectado en este grupo fue de 6 (media de 5,4). La evolución fue favorable en todos ellos. Ningún paciente al que se le realizó RT progresó posteriormente durante el periodo de seguimiento, tras una media de seguimiento de 37,7 meses (rango 18-72).

El manejo de las progresiones fue diverso: 3 pacientes recibieron RT externa, uno de ellos con tratamiento hormonal previo; un paciente recibió braquiterapia; y 6 pacientes recibieron únicamente tratamiento hormonal. En 2 pacientes de este último grupo la progresión continuó a pesar de diversas pautas de tratamientos hormonales y terapias de segunda y tercera línea. La evolución fue favorable en 8 de los 10 casos sin datos de progresión posterior tras una media de seguimiento a partir de la detección y tratamiento de la progresión de 34,4 meses (rango 12-72).

La mortalidad global CPI específica a lo largo del periodo de seguimiento $(47,19$ meses, rango 12-96) fue del $2,6 \%$ ( 2 de 75 pacientes). La mortalidad CPI específica en el grupo de progresión (10 casos) fue del $20 \%$ ( 2 de 10 pacientes). Los dos pacientes fallecidos por progresión del cáncer de próstata pertenecían al grupo de las RTU-P; el CPI detectado fue manejado en ambos casos mediante tratamiento hormonal; y fueron tratados de la progresión de la enfermedad mediante diversas pautas y variaciones del bloqueo hormonal y terapias de $2^{\underline{a}}$ y $3^{\text {a }}$ línea sin éxito.

Durante el periodo de seguimiento de los pacientes con CPI se detectaron además otras neoplasias que condicionaron en muchos de los casos la evolución de dichos sujetos. Este "indice de otros tumores" en estos pacientes fue elevado, probablemente condicionado por los factores genéticos-familiares y ambientales que condicionan la aparición de las neoplasias. En un total de 18 pacientes de 75 se detectaron otros tumores malignos durante el periodo de seguimiento (24\% de la población de pacientes con CPI), predominando los de vejiga (9 casos), los digestivos (4 casos), los hematológicos (3 casos), de origen ORL (1 caso), y dermatológicos (1 caso). 


\section{COMENTARIOS}

En un interesante artículo americano ${ }^{6}$, se determinó si los pacientes que se iban a someter a cistoprostatectomía radical por cáncer vesical infiltrante tenían alta incidencia de cáncer prostático no sospechado (incidental). A pesar de que la serie de pacientes era pequeña (40), en el $45 \%$ de ellos se detectó tumor prostático tras el análisis de la pieza quirúrgica. Dada esta alta incidencia (condicionada por la edad elevada de los pacientes), se recomienda como parte del estudio previo a la cirugía y al manejo de los tumores infiltrantes vesicales, el tacto rectal, PSA y una exéresis completa de la próstata en el procedimiento quirúrgico.

En series publicadas importantes, la prevalencia de CPI oscila según los distintos tipos de trabajos entre el 3,4 - 13\% $\%^{1-5}$. Incluso el PSA no se muestra como un buen predictor de $\mathrm{CPI}^{4}$. En un trabajo destacable $^{7}$, sobre una serie de pacientes importante (6.426), se analizó la prevalencia de CPI en RTU-P a lo largo de diversos periodos de tiempo (39\% entre 1980-1984, 34\% entre 1985-1989, 12\% entre 1990-1994, 7,4\% entre 1995-1999), observando el descenso paulatino a medida que nos acercamos a la actualidad por el incremento en el diagnóstico precoz del cáncer prostático.

Las progresiones tumorales y la mortalidad CPI específica también son variables según los trabajos revisados, dependiendo de los pacientes y de los tratamientos ${ }^{8-10}$. Incluso se ha defendido una segunda RTU-P (second-look) para definir el estadio tumoral y decidir el tratamiento ${ }^{11}$.

En nuestra serie de PR tras el diagnóstico de CPI, 6 de los 7 casos presentaron tumor al analizar la pieza quirúrgica y sólo se evidenció un caso con estadio final pTO. Esta alta incidencia de tumor residual y el riesgo que conlleva, ha hecho que numerosos autores recomienden un tratamiento curativo en pacientes jóvenes con expectativa de vida superior a los 10-15 años ${ }^{2,8}$.

\section{CONCLUSIONES}

Tras revisión y análisis de los resultados obtenidos en el estudio, no observamos diferencias significativas entre la prevalencia de CPI (media de 4,89\%) entre el grupo de las supuestas HBP operadas mediante APS $(4,55 \%)$ y las operadas mediante RTU-P (5,07\%). Los tumores incidentales detectados fueron mayoritariamente bien diferenciados, con una mediana del grado de Gleason de 5 (media 4,8) y en estadio pTla $(66,6 \%)$. En más de la mitad de los casos $(57,7 \%)$ se decidió una actitud expectante sin tratamiento. La progresión tumoral global fue del 13,3\% (10 de 75), $12 \%$ en el grupo APS ( 3 de 25 ) y 14\% en el grupo RTU-P (7 de 50), tras 47,19 meses de seguimiento medio (rango 12-96 meses). La mortalidad global específica del CPI fue del 2,6\% (2 de 75), y del $20 \%$ en el grupo de progresión tumoral ( 2 de 10 ).

\section{REFERENCIAS}

1. Picurelli Oltra L, Sendra Torres A, Fernández Rodríguez A, Ortega Villar F, Baixauli Martinez J, Tramoyeres Celma A. Incidental prostatic adenocarcinoma in the era of the PSA. Actas Urol Esp 1997;21:354-356.

2. Van Andel G, Vleeming R, Kurth K, de Reijke TM. Incidental carcinoma of the prostate. Semin Surg Oncol 1995; 11:36-45.

3. Herranz Amo F, Díez Cordero JM, Verdú Tartajo F, Leal Hernández F, Bielsa Carrillo A, García Burgos J, et al. Incidental prostatic cancer. Actas Urol Esp 1999;23:848-852.

4. Mai KT, Isotalo PA, Green J, Perkins DG, Morash C, Collins JP. Incidental prostatic adenocarcinomas and putative premalignant lesions in TURP specimens collected before and after the introduction of PSA screening. Arch Pathol Lab Med 2000;124:1454-1456.

5. Zigeuner RE, Lipsky K, Riedler I, Auprich M, Schips L, Salfellner M, et al. Did the rate of incidental prostate cancer change in the era of PSA testing? A retrospective study of 1.127 patients. Urology 2003;62:451-455.

6. Abbas F, Hochberg D, Civantos F, Soloway M. Incidental prostatic adenocarcinoma in patients undergoing radical cystoprostatectomy for bladder cancer. Eur Urol 1996;30: 322-326.

7. Merrill RM, Wiggins CL. Incidental detection of populationbased prostate cancer incidence rates through tranurethral resection of the prostate. Urol Oncol 2002;7:213-219.

8. Schurholz T, Schulze H, Finke W, Lange S, Senge T. Clinical relevante of incidental prostate carcinoma. A retrospective study. Urologe A 1996;35:315-320.

9. Cheng L, Bergstralh EJ, Scherer BG, Neumann RM, Blute ML, Zincke H, et al. Predictors of cancer progresión in Tla prostate adenocarcinoma. Cancer. 1999,85:1300-1304.

10. Nuñez López AV, Ojea Calvo A, Domínguez Freire F, Alonso Rodrigo AE, Rodríguez Iglesias B, Benavente Delgado J, et al. Clinical and biological progresión of incidental prostatic cancer (stage T1a). Actas Urol Esp 2003;27:345-349.

11. Ford TF, Cameron KM, Parkinson MC, O`Donoghue EP. Incidental carcinoma of the prostate: treatment selection by second-look TURP. Br J Urol 1984;56:682-686.

Dr. E. Fernández Rosado

E-mail: efrosado@ozu.es

(Trabajo recibido el 27 de mayo 2005) 